\title{
Critical seismic load inputs for simple inelastic structures
}

\author{
A. M. Abbas* \\ Department of Civil Engineering, Faculty of Engineering, Minia University, Minia \\ 61111, Egypt
}

\begin{abstract}
The modelling of earthquake loads as design inputs for inelastic single-degree-offreedom structures is considered. The earthquake load is modelled as a deterministic time history which is expressed in terms of a Fourier series that is modulated by an enveloping function. Subsequently, the coefficients of the series representation, and, the parameters of the envelope function are determined such that the structure inelastic deformation is maximized subject to a set of predefined constraints. These constraints include bounds on the total energy of the earthquake signal, peak values on ground acceleration, velocity and displacement and upper and lower bounds on the Fourier spectra of the ground acceleration. Additional mathematical limits on the envelope parameters are also considered. The quantification of these constraints is obtained based on numerical analysis of a set of past recorded ground motions at the site under consideration or other sites with similar soil conditions. The structure force-displacement relation is taken to possess an elastic-plastic behavior. The resulting nonlinear optimization problem is tackled by using the sequential quadratic optimization method. The study, also, examines influences of the structure yield strength and damping ratio on the derived earthquake load and the associated structure response. Issues related to the time-variation of various energy forms dissipated by the inelastic system are also explored. The proposed formulation is demonstrated with reference to the inelastic response analysis of a frame structure driven by a single component of earthquake load.
\end{abstract}

Key words: Earthquake loads, inelastic structures, ductility factor, dissipated energy, dynamic response, nonlinear optimization

\footnotetext{
* Visiting faculty, Dept. of Civil Eng., Faculty of Eng., Nagasaki University, Nagasaki 852-8521, Japan

Email address: abbas@civil.nagasaki-u.ac.jp (A. M. Abbas).
} 


\section{Introduction}

The problem of modelling earthquake ground motions as design inputs for engineering structures has received significant research attention worldwide. The present practice is to use the method of design response spectra, the time history analysis or the method of random vibration. The recent paper by Bommer and Acevedo [1] provides a critical review on the use of real earthquake accelerograms as inputs to dynamic analysis of engineering structures. On the other hand, the method of critical earthquake load modelling has been established, during the last three decades, as a counterpart to these methods. This method relies on the fact that, for many parts of the world, available data on strong earthquake ground motion is either inhomogeneous or insufficient. The method of critical earthquake loads is a powerful tool for predicting forthcoming earthquake accelerations for sites lacking information on earthquake ground motion. It may be noted that one of the important aspects which contributes significantly to damage of structures is the resonant nature of earthquake ground motion. This feature has been observed in actual recorded ground motions. For instance, the Fourier spectra of the first horizontal component of the San Fernando 1971 earthquake and Hyogoken-Nanbu 1995 Japan earthquake are shown in figure (1). It is obvious from this figure that both accelerograms exhibit resonant nature at very few frequencies. The influence of the site soil characteristics, in terms of dominant frequency, Fourier amplitude and frequency bandwidth of the ground motion is apparent in each of these records. Structures having their fundamental natural frequency close to the dominant frequency of any of these accelerograms can be expected to produce high responses. The method of critical earthquake excitations provides the worst case scenario that can happen to the structure at a given site. Accordingly, this method accounts for the uncertainties associated with the specification process of earthquake loads as design inputs for structures. On the other hand, it is well known that modeling structural nonlinear behavior is of central importance in earthquake engineering. It is, thus, of interest to investigate the development of the method of critical earthquake load modelling to structures deforming into inelastic stage. This is particularly true when dealing with dynamic analysis of structures driven by extreme loads as is the case with critical earthquake loads.

Early works on modelling critical earthquake loads has been carried out by Drenick [2], Shinozuka [3] and Iyengar [4]. An extensive overview of the development of this method is reported by Takewaki [5], Abbas and Manohar [6] and Abbas [7]. This method can be developed within deterministic or probabilistic frameworks. In the deterministic approach, the earthquake load is defined as an acceleration time history or in terms of response spectra. In the probabilistic approach, the earthquake ground motion is modelled as a random process. Regardless of the framework adopted, critical earthquake loads 
depend upon the structure considered, the site soil conditions beneath the structure and the constraints imposed on the earthquake signal. In implementing this method, the earthquake load is taken to be known only partially and an inverse dynamic problem is solved to compute the unknown information on the seismic input, such that, a pre-selected damage variable of the structure is maximized. At the same time the computed load, termed as 'critical' excitation, satisfies a set of constraints that impart known features of real earthquake ground motion. Drenick [2] was the first to introduce the method of critical excitations to the field of earthquake engineering within the framework of deterministic analysis. He considered maximizing the dynamic response of linear single-degree-of-freedom systems driven by ground acceleration and showed that the critical excitation for such systems, under a constraint on total energy, is the impulse response function reversed in time. Shinozuka [3] improved Drenick's approach by introducing an upper bound on the Fourier transform of the ground acceleration. Iyengar [4] incorporated the non-stationarity trend and total duration of recorded ground acceleration in deriving critical earthquake loads. The modelling of random critical earthquake loads, for linear structures, was introduced by Iyengar and Manohar [8]. These authors modelled the ground acceleration as a non-stationary Gaussian random process by multiplying a known envelope function by a stationary random process of unknown power spectral density function. They obtained the power spectral density function of the stationary part by maximizing the structure response variance under a constraint on the average total energy of the input. Abbas and Manohar [6] modelled critical earthquake loads for linear structures within deterministic and probabilistic frameworks. These authors investigated the realism of new proposed constraints in each framework. This study showed that the constraints on upper and lower bounds on the Fourier spectra of the ground acceleration in the deterministic framework and the constraint on the entropy rate in the probabilistic model are crucial in developing realistic critical earthquake loads.

While the problem of modelling critical earthquake loads for linear structures is widely studied, the determination of critical earthquake excitations for nonlinear structures, on the other hand, has been studied to a very limited extent in the existing literature. Given that the treatment of structure nonlinearities is of central importance in earthquake-resistant design, it is, thus, of significant interest to develop methods for computing critical earthquake excitations for structures deforming into their inelastic range. Iyengar [9] studied the problem of deriving critical earthquake loads for a class of nonlinear single-degree-of-freedom systems by imposing a constraint on the input total energy. The force-deformation relation, in this study, was modelled using Duffing oscillators. Drenick [10] extended his earlier study on linear structures to nonlinear structures using equivalent linearization. He showed that the critical excitation for a nonlinear system is again, except for a constant factor, the time reversed impulse response function of the linearized system. Philip- 
pacopoulos and Wang [11] developed critical inelastic response spectra using recorded ground accelerograms as basis functions in a series representation for the critical seismic excitation. Westermo [12] defined critical response in terms of input energy to the system and determined critical excitations for elastic-plastic and hysteretic single-degree-of-freedom systems using calculus of variations. For linear systems he showed that critical inputs are harmonic and derivable from the harmonically excited response functions of the system. On the other hand, critical loads for inelastic systems were not harmonic and at low frequencies the response is significantly larger than the harmonically excited response. Notwithstanding this, the critical excitations computed in these studies do not possess realistic characteristics of actual earthquake loads, and, thus cannot be considered as realistic models for earthquake accelerograms. A similar study to that reported by Westermo was carried out by Pirasteh et al., [13]. These authors computed the critical excitations for inelastic multi-story frame structures under deterministic earthquake inputs. The response variable adopted for maximization was chosen as the cumulative inelastic energy dissipation or sum of inter-story drifts. The objective functions, in this study, were evaluated using approximate methods to reduce the computational costs of the nonlinear dynamic response analysis. Recently, Takewaki $[14,15]$ has developed critical input power spectral density function models for earthquake inputs to single-degree-of-freedom and multi-degree-offreedom elastic-plastic systems. This author utilizes the method of statistical linearization to approximately evaluate the structure response. The variable of optimization in these two studies has been the sum of the response standard deviations of inter-story drifts normalized to yield drifts. More recently, Abbas and Manohar [16] have developed a reliability-based framework for determining random critical earthquake loads for nonlinear structures. This study integrates methods of structural reliability analysis, response surface modelling and nonlinear programming in computing seismic inputs for structures having cubic force-displacement relations. The damage variable was adopted as the structure reliability index.

The present study addresses the problem of modelling critical earthquake inputs for inelastic structures. The earthquake load is modelled as a deterministic time history which is expressed in terms of a Fourier series, of unknown coefficients, that is modulated by an enveloping function. While earlier studies on modelling deterministic critical earthquake loads (Abbas and Manohar [6] and Abbas [7]) treat the envelope function as being a known function, the present study, on the other hand, considers the envelope parameters as unknown quantities to be optimally determined. The coefficients of the series representation and the parameters of the envelope function are determined such that the structure inelastic response is maximized subject to a set of predefined constraints. The constraints invoked on the earthquake signal are taken to reflect known characteristics of actual recorded ground motions. Specifically, the constraints include upper bounds on the total earthquake energy and peak 
values of ground acceleration, velocity and displacement. Upper and lower limits on the Fourier spectra of the ground acceleration are also considered. The constraints, also, include mathematical bounds on the parameters of the envelope function. The structure force-displacement relation is taken to possess elastic-plastic characteristic. The resulting nonlinear optimization problem is solved by using the sequential quadratic optimization method. Since, to the best of author's knowledge the influence of yield strength and damping ratio on derived critical earthquake excitations has not been studied earlier, the present study examines these aspects. The study, also, tackles issues related to dissipated energy by the inelastic system. Numerical illustrations on modelling critical earthquake loads for an elastic-plastic frame structure located at a firm soil site are provided.

\section{Seismic response of inelastic single-degree-of-freedom structures}

The equation of motion governing the relative displacement response $u(t)$ of a single-degree-of-freedom system subject to a single component of earthquake ground motion $\ddot{u}_{g}(t)$ (see figure $2(\mathrm{a})$ ) is well known to be given by

$$
m \ddot{u}(t)+c \dot{u}(t)+f_{s}(t)=-m \ddot{u}_{g}(t)
$$

Here, $m, c$, are, respectively, the mass and damping of the single-degree-offreedom system and $f_{s}(t)$ is the restoring force in the spring. The above equation of motion may describe the dynamic response analysis of a single-storey frame structure or a piping system under a uniform ground motion $\ddot{u}_{g}(t)$. For linear structural behavior the restoring force $f_{s}(t)$ is a linear function of the displacement response $u(t)$ and the spring stiffness coefficient $k$. Whereas, in the more general case, when structural nonlinearities are considered, this force is a nonlinear function of the structure response. For instance, figure 2(b) depicts the nature of $f_{s}(t)$ for nonlinear systems with force-displacement characteristic modelled using elastic-plastic behavior. Herein, the restoring force is not only a function of the displacement response but depends on the velocity response as well. Therefore, for nonlinear dynamical systems with elastic-plastic characteristics, the above equation of motion may be re-written as

$$
m \ddot{u}(t)+c \dot{u}(t)+f_{s}(u, \dot{u})=-m \ddot{u}_{\mathrm{g}}(t)
$$

It may be noted that, for systems governed by the above equation of motion, the force-deformation relation is no longer a single valued relation. Thus, for a displacement $u\left(t_{i}\right)$ at time $t_{i}$ the resisting force depends upon prior history of motion of the system and whether velocity response $\dot{u}\left(t_{i}\right)$ is increasing or decreasing. In the present work, damping is taken to be viscous, and, also, it 
is assumed that system starts from rest. While, it is possible to treat damping as being nonlinear, the present study, however, does not consider this aspect. It is intended in this study to focus on the complicating features arising due to inelastic structural behavior. The above equation of motion can be recast as

$$
\ddot{u}(t)+2 \zeta_{n} \omega_{n} \dot{u}(t)+\omega_{n}^{2} u_{y} \bar{f}_{s}(u, \dot{u})=-\ddot{u}_{\mathrm{g}}(t)
$$

where, $\zeta_{n}=c / 2 \sqrt{k m}$ is the damping ratio, $\omega_{n}=\sqrt{k / m}$ is the natural frequency for the linear system or for the elastic-plastic system undergoing small deformations (i.e. $u \leq u_{y}$ ) and $u_{y}$ is the yield displacement. It may be recalled that, at larger amplitudes the natural vibration period is not defined for inelastic systems. The function $\bar{f}_{s}(u, \dot{u})$ may be defined as the spring restoring force in a dimensionless form. Refereing to the above equation, it may be noted that for a given earthquake acceleration $\ddot{u}_{g}(t)$, the displacement response depends on the natural frequency $\omega_{n}$, the damping ratio $\zeta_{n}$ and the yield displacement $u_{y}$ (see figure 2(b)). Herein, the yield displacement $u_{y}$ is defined as $f_{y} / k$ where $f_{y}$ is the yield strength. The dynamic analysis of inelastic structures governed by the above equation of motion can be carried out directly by solving this equation. Alternatively, the dynamic analysis of these systems can be characterized in terms of the inelastic displacement response normalized to the yield displacement. This dimensionless quantity is known as the ductility factor. Thus, defining this factor as $\mu(t)=u(t) / u_{y}$ and substituting into equation (3) one gets

$$
\ddot{\mu}(t)+2 \zeta_{n} \omega_{n} \dot{\mu}(t)+\omega_{n}^{2} \bar{f}_{s}(\mu, \dot{\mu})=-\omega_{n}^{2} \frac{\ddot{u}_{\mathrm{g}}(t)}{a_{y}}
$$

It follows from this equation of motion that the ductility factor for systems driven by a time-variant dynamical load is also a time-variant quantity. It may be observed that the expressions $\ddot{u}(t)=u_{y} \ddot{\mu}(t)$ and $\dot{u}(t)=u_{y} \dot{\mu}(t)$ were employed in deriving the above equation. The constant $a_{y}=f_{y} / m$, appearing in the right side of this equation, can be interpreted as the acceleration of the mass necessary to produce the yield force $f_{y}$ and $\bar{f}_{s}(\mu, \dot{\mu})$ is the forcedeformation relation in dimensionless form. Furthermore, the acceleration ratio $\frac{\ddot{u}_{g}(t)}{a_{y}}$ is the ratio between the ground acceleration and a measure of the yield strength of the structure. For instance, equation (4) implies that doubling the ground acceleration $\ddot{u}_{g}(t)$ will produce the same response $\mu(t)$ as if the yield strength had been halved. The response analysis of inelastic systems governed by the above equation of motion (or equation 3 ) is generally carried out using numerical integration techniques. 


\section{Critical earthquake loads for inelastic single-degree-of-freedom structures}

The formulation for modelling critical earthquake excitations for elastic-plastic single-degree-of-freedom structures is presented in this section. As a first step, the ground acceleration appearing in the right side of equation (4) is represented as a product of a Fourier series and an enveloping function as follows

$$
\ddot{u}_{g}(t)=e(t) \quad\left[\sum_{i=1}^{N_{f}} A_{i} \cos \omega_{i} t+B_{i} \sin \omega_{i} t\right]
$$

Here, $A_{i}, B_{i}$, are $2 N_{f}$ unknown constants and $\omega_{i}, i=1,2, \ldots, N_{f}$, are the frequencies presented in the ground acceleration $\ddot{u}_{g}(t)$ which are selected such that they span satisfactory the frequency range $\left(\omega_{0}, \omega_{c}\right)$ of $\ddot{u}_{g}(t)$. The function $e(t)$ represents the enveloping function that imparts transient nature to the earthquake acceleration. In the present study, the envelope function $e(t)$ is taken to be given by [17]

$$
e(t)=\frac{\exp \left(-\alpha_{1} t\right)-\exp \left(-\alpha_{2} t\right)}{\max \left[\exp \left(-\alpha_{1} t\right)-\exp \left(-\alpha_{2} t\right)\right]} ; \quad \alpha_{2}>\alpha_{1}>0
$$

Herein, $\alpha_{1}$ and $\alpha_{2}$ are the parameters of the enveloping function. The maximum value of the envelope function as per the above expression is unity. It may be recalled that earlier studies [6,7] have considered these parameters as known variables. In the present study, these parameters are treated as unknown quantities to be optimally determined. Accordingly, in constructing critical earthquake excitations for elastic-plastic systems, it is assumed that $A_{i}, B_{i}, i=1,2, \ldots, N_{f}, \alpha_{1}$ and $\alpha_{2}$ are unknowns. Furthermore, the information on energy $E$, peak ground acceleration (PGA) $M_{1}$, peak ground velocity (PGV) $M_{2}$, peak ground displacement (PGD) $M_{3}$, upper bound Fourier amplitude spectra (UBFAS) $M_{4}(\omega)$ and lower bound Fourier amplitude spectra (LBFAS) $M_{5}(\omega)$ are taken to be available which enables the formulation of the following constraints

$$
\begin{array}{r}
{\left[\int_{0}^{\infty} \ddot{u}_{g}^{2}(t) d t\right]^{1 / 2} \leq E ;} \\
\max _{0<t<\infty}\left|\ddot{u}_{g}(t)\right| \leq M_{1} ; \\
\max _{0<t<\infty}\left|\dot{u}_{g}(t)\right| \leq M_{2} ; \\
\max _{0<t<\infty}\left|u_{g}(t)\right| \leq M_{3} ; \\
M_{5}(\omega) \leq V_{g}(\omega) \leq M_{4}(\omega)
\end{array}
$$


The function $V_{g}(\omega)$ appearing in the above equation represents the Fourier transform of the ground acceleration $\ddot{u}_{g}(t)$. In addition to the above set of constraints, mathematical bounds on the parameters of enveloping function are also considered. These constraints are given as

$$
\begin{gathered}
\alpha_{1 l} \leq \quad \alpha_{1} \leq \alpha_{1 u} \\
\alpha_{2 l} \leq \quad \alpha_{2} \leq \alpha_{2 u}
\end{gathered}
$$

It may be remarked that the positivity condition on $\alpha_{1}$ and $\alpha_{2}$ (see equation 6 ) is implicitly satisfied if the above bounds are appropriately selected. To proceed further, the ground velocity and displacement should be computed. Thus, making use of equation (5), one gets

$$
\begin{aligned}
& \dot{u}_{g}(t)=\sum_{i=1}^{N_{f}} \int_{0}^{t} e(\tau)\left[A_{i} \cos \omega_{i} \tau+B_{i} \sin \omega_{i} \tau\right] d \tau+C_{1} \\
& u_{g}(t)=\sum_{i=1}^{N_{f}} \int_{0}^{t} e(\tau)(t-\tau)\left[A_{i} \cos \omega_{i} \tau+B_{i} \sin \omega_{i} \tau\right] d \tau+C_{1} t+C_{2}
\end{aligned}
$$

The constants $C_{1}$ and $C_{2}$ appearing above are found using the conditions [18]

$$
u_{g}(0)=0, \quad \lim _{t \rightarrow \infty} \dot{u}_{g}(t) \rightarrow 0
$$

This leads to

$$
C_{2}=0, \quad C_{1}=-\sum_{i=1}^{N_{f}} \int_{0}^{\infty} e(\tau)\left[A_{i} \cos \omega_{i} \tau+B_{i} \sin \omega_{i} \tau\right] d \tau
$$

Subsequently, the constraints listed in equations (7) and (8) can be expressed in terms of the unknown variables $A_{n}, B_{n}, n=1,2, \ldots, N_{f}, \alpha_{1}$ and $\alpha_{2}$ as follows

$$
\begin{gathered}
{\left[\sum_{m=1}^{N_{f}} \sum_{n=1}^{N_{f}} A_{m} A_{n} I_{1}\left(\omega_{m}, \omega_{n}\right)+A_{m} B_{n} I_{2}\left(\omega_{m}, \omega_{n}\right)+B_{m} A_{n} I_{3}\left(\omega_{m}, \omega_{n}\right)+B_{m} B_{n} I_{4}\left(\omega_{m}, \omega_{n}\right)\right]^{1 / 2} \leq E ;} \\
\quad \max _{0<t<\infty}\left|\frac{\exp \left(-\alpha_{1} t\right)-\exp \left(-\alpha_{2} t\right)}{\max \left[\exp \left(-\alpha_{1} t\right)-\exp \left(-\alpha_{2} t\right)\right]} \sum_{n=1}^{N_{f}}\left[A_{n} \cos \omega_{n} t+B_{n} \sin \omega_{n} t\right]\right| \leq M_{1} ; \\
\max _{0<t<\infty} \mid \sum_{n=1}^{N_{f}} \int_{0}^{t} \frac{\exp \left(-\alpha_{1} \tau\right)-\exp \left(-\alpha_{2} \tau\right)}{\max \left[\exp \left(-\alpha_{1} \tau\right)-\exp \left(-\alpha_{2} \tau\right)\right]}\left[A_{n} \cos \omega_{n} \tau+B_{n} \sin \omega_{n} \tau\right] d \tau \\
-\sum_{n=1}^{N_{f}} \int_{0}^{\infty} \frac{\exp \left(-\alpha_{1} \tau\right)-\exp \left(-\alpha_{2} \tau\right)}{\max \left[\exp \left(-\alpha_{1} \tau\right)-\exp \left(-\alpha_{2} \tau\right)\right]}\left[A_{n} \cos \omega_{n} \tau+B_{n} \sin \omega_{n} \tau\right] d \tau \mid \leq M_{2} ;
\end{gathered}
$$




$$
\begin{array}{r}
\max _{0<t<\infty} \mid \sum_{n=1}^{N_{f}} \int_{0}^{t} \frac{\exp \left(-\alpha_{1} \tau\right)-\exp \left(-\alpha_{2} \tau\right)}{\max \left[\exp \left(-\alpha_{1} \tau\right)-\exp \left(-\alpha_{2} \tau\right)\right]}(t-\tau)\left[A_{n} \cos \omega_{n} \tau+B_{n} \sin \omega_{n} \tau\right] d \tau \\
-t \sum_{n=1}^{N_{f}} \int_{0}^{\infty} \frac{\exp \left(-\alpha_{1} \tau\right)-\exp \left(-\alpha_{2} \tau\right)}{\max \left[\exp \left(-\alpha_{1} \tau\right)-\exp \left(-\alpha_{2} \tau\right)\right]}\left[A_{n} \cos \omega_{n} \tau+B_{n} \sin \omega_{n} \tau\right] d \tau \mid \leq M_{3} \\
M_{5}(\omega) \leq\left|\sum_{n=1}^{N_{f}} A_{n} I_{1 n}(\omega)+B_{n} I_{2 n}(\omega)\right| \leq M_{4}(\omega) ; \\
\alpha_{1 l} \leq \alpha_{1} \leq \alpha_{1 u} ; \\
\left.\alpha_{2 l} \leq \alpha_{2} \leq \alpha 6 t_{l} 3\right)
\end{array}
$$

where

$$
\begin{gathered}
I_{1}\left(\omega_{m}, \omega_{n}\right)=\int_{0}^{\infty}\left[\frac{\exp \left(-\alpha_{1} t\right)-\exp \left(-\alpha_{2} t\right)}{\max \left[\exp \left(-\alpha_{1} t\right)-\exp \left(-\alpha_{2} t\right)\right]}\right]^{2} \cos \omega_{m} t \cos \omega_{n} t d t \\
I_{2}\left(\omega_{m}, \omega_{n}\right)=\int_{0}^{\infty}\left[\frac{\exp \left(-\alpha_{1} t\right)-\exp \left(-\alpha_{2} t\right)}{\max \left[\exp \left(-\alpha_{1} t\right)-\exp \left(-\alpha_{2} t\right)\right]}\right]^{2} \cos \omega_{m} t \sin \omega_{n} t d t \\
I_{3}\left(\omega_{m}, \omega_{n}\right)=\int_{0}^{\infty}\left[\frac{\exp \left(-\alpha_{1} t\right)-\exp \left(-\alpha_{2} t\right)}{\max \left[\exp \left(-\alpha_{1} t\right)-\exp \left(-\alpha_{2} t\right)\right]}\right]^{2} \sin \omega_{m} t \cos \omega_{n} t d t ; \\
I_{4}\left(\omega_{m}, \omega_{n}\right)=\int_{0}^{\infty}\left[\frac{\exp \left(-\alpha_{1} t\right)-\exp \left(-\alpha_{2} t\right)}{\max \left[\exp \left(-\alpha_{1} t\right)-\exp \left(-\alpha_{2} t\right)\right]}\right]^{2} \sin \omega_{m} t \sin \omega_{n} t d t ; \\
I_{1 n}(\omega)=\int_{0}^{\infty} \frac{\exp \left(-\alpha_{1} t\right)-\exp \left(-\alpha_{2} t\right)}{\max \left[\exp \left(-\alpha_{1} t\right)-\exp \left(-\alpha_{2} t\right)\right]} \cos \omega_{n} t \exp (-j \omega t) d t ; \\
\left.I_{2 n}(\omega)=\int_{0}^{\infty} \frac{\exp \left(-\alpha_{1} t\right)-\exp \left(-\alpha_{2} t\right)}{\max \left[\exp \left(-\alpha_{1} t\right)-\exp \left(-\alpha_{2} t\right)\right]} \sin \omega_{n} t \exp (-j \omega t) d t ; j=\sqrt{-\mathbb{1} 4}\right)
\end{gathered}
$$

To determine the quantities $E, M_{1}, M_{2}, M_{3}, M_{4}(\omega)$ and $M_{5}(\omega)$ it is assumed that a set of earthquake records denoted by $\left\{\ddot{v}_{g i}(t)\right\}_{i=1}^{N_{r}}$ are available for the site under consideration or from other sites that are geologically similar to the given site. The values of intensity, peak values of acceleration, velocity and displacement are obtained for each of these records. The highest of these values across the ensemble of the records are taken to be the respective estimates of $E, M_{1}, M_{2}$ and $M_{3}$. The set of available records $\left\{\ddot{v}_{g n}(t)\right\}_{n=1}^{N_{r}}$ are further normalized such that the energy of each record is set to unity, and these normalized records are denoted by $\left\{\ddot{\bar{v}}_{g i}(t)\right\}_{i=1}^{N_{r}}$. The bounds $M_{4}(\omega)$ and $M_{5}(\omega)$ are obtained as

$$
\begin{aligned}
& M_{4}(\omega)=E \max _{1 \leq i \leq N_{r}}\left|\bar{V}_{g i}(\omega)\right| \\
& M_{5}(\omega)=E \min _{1 \leq i \leq N_{r}}\left|\bar{V}_{g i}(\omega)\right|
\end{aligned}
$$


Here, the function $\bar{V}_{g i}(\omega), i=1,2, \ldots, N_{r}$ denotes the Fourier transform of the $i$ th normalized accelerogram $\ddot{\bar{v}}_{g i}(t)$ and these transforms are computed using the Fast Fourier Transform. It may be noted that the idea of introducing an upper bound on the Fourier amplitude of the ground motion has been considered earlier by Shinozuka [3], Baratta et al., [19] and, also, in a probabilistic setting, by Takewaki $[14,15]$. On the other hand, the lower bound on the Fourier amplitude spectra was considered in reference [6]. It may also be remarked that the assumption on availability of past records $\left\{\ddot{v}_{g n}(t)\right\}_{n=1}^{N_{r}}$ is similar to the assumption made by Drenick [20]. While the earlier workers [11, 20,21] employed these records as basis functions, in this study, these records are used to derive the constraints that the critical excitations need to satisfy. The quantification of the bounds of the parameters of the enveloping function $\alpha_{1 l}, \alpha_{1 u}, \alpha_{2 l}$ and $\alpha_{2 u}$ are made available based on a numerical analysis of the set of past recorded accelerograms $\ddot{v}_{g i}(t), i=1,2, \ldots, N_{r}$. To quantify these constraint limits, each earthquake accelerogram $\ddot{v}_{g i}(t), i=1,2, \ldots, N_{r}$ is assumed to be represented by the form of equation (5). The associated envelope function to this record is taken to be given by equation (6). Subsequently, the parameters of the envelope function $\alpha_{1}$ and $\alpha_{2}$ are determined such that they match the transient trend of the ground acceleration. The constraint bounds $\alpha_{1 u}$ and $\alpha_{2 u}$ are computed as the highest values across the ensemble $\left\{\alpha_{1 i}, \alpha_{2 i}\right\}_{i=1}^{N_{r}}$, respectively. Similarly, $\alpha_{1 l}$ and $\alpha_{2 l}$ are taken as the lowest values of $\left\{\alpha_{1 i}, \alpha_{2 i}\right\}_{i=1}^{N_{r}}$, respectively.

Finally, the problem of deriving critical earthquake loads for elastic-plastic structures can be posed as determining the optimization variables $x=\left[A_{1}\right.$, $\left.A_{2}, \ldots, A_{N_{f}}, B_{1}, B_{2}, \ldots, B_{N_{f}}, \alpha_{1}, \alpha_{2}\right]^{t}$ such that the structure inelastic response (as given in equation (4)) is maximized subject to the constraints listed in equations (13). This constitutes a constrained nonlinear optimization problem and is tackled by using the Sequential Quadratic Programming (SQP) method [22]. Herein, the optimization problem is solved iteratively starting from a pre-specified initial guess for the vector of the optimization variables $x$. Subsequently, the optimization algorithm performs a sensitivity analysis, at each iteration, searching for new values for the vector of the optimization variables $x$. The optimization code converges to the optimal solution when the following criteria on the objective function and optimization variables are satisfied

$$
\begin{array}{r}
\left|\mu_{j}-\mu_{j-1}\right| \leq \ell_{1} \\
\left|x_{i j}-x_{i-1 j}\right| \leq \ell_{2} ; \quad i=1,2, \ldots, N_{f}+2
\end{array}
$$

Herein, $j$ represents the iteration number and $x_{i j}$ indicates the $i$ th optimization variable at the $j$ th iteration. The structure inelastic response is determined via numerical integration of the equation of motion by using the Newmark- $\beta$ method. The details of the steps involved in the computation of the optimal 
earthquake loads and the corresponding inelastic response of the structure can be summarized as follows

(1) Define the structure parameters $m, c, k$, the yield strength in tension and compression $\left(f_{y t} \& f_{y c}\right)$ and determine the parameter $a_{y}=\frac{f_{y t}}{m}$.

(2) Set the initial conditions $\mu(0)$ and $\dot{\mu}(0)$ and compute the corresponding quantity $\ddot{\mu}(0)$ from the equilibrium of the equation of motion (4). Herein, the initial conditions $u(0)=0$ and $\dot{u}(0)=0$ and the transformation $\mu(t)=\frac{u(t)}{u_{y t}}$ are employed in determining $\mu(0)$ and $\dot{\mu}(0)$.

(3) Select the time step $\Delta t$ and calculate the constants of the Newmark- $\beta$ method $\left(a_{1}=\frac{1}{\beta \Delta t}, a_{2}=\frac{1}{2 \beta}, a_{3}=1-\frac{1}{4 \beta}, a_{4}=\frac{1}{\beta \Delta t^{2}}\right)$.

(4) Determine the initial yield ductility points $\mu_{y t}=\frac{\left.u(t)\right|_{t=t_{y t}}}{u_{y t}}=1$ and $\mu_{y c}=$ $\frac{\left.u(t)\right|_{t=t_{y c}}}{\left|u_{y c}\right|}=-1$. Here, $t_{y t}$ and $t_{y c}$ define the time points at which system starts to yield in tension and in compression, respectively.

(5) For $t=t_{j}$ use the value of the parameter KEY to establish the elastic or plastic state of the structure based on the following criteria

- $\mathrm{KEY}=0$ implies elastic behavior

- $\mathrm{KEY}=1$ implies plastic behavior in tension

- $\mathrm{KEY}=-1$ implies plastic behavior in compression

(6) Calculate the incremental effective force $\Delta \bar{F}_{j}=-\frac{\omega_{n}^{2}}{a_{y}} \Delta \ddot{u}_{g j}+\left(a_{1}+2 a_{2} \zeta_{n}\right.$ $\left.\omega_{n}\right) \dot{\mu}_{j}+\left(a_{2}-2 a_{3} \zeta_{n} \omega_{n}\right) \ddot{\mu}_{j}$

(7) Calculate the effective stiffness $\bar{K}_{j}=\omega_{n}^{2} k_{p}+a_{1} \zeta_{n} \omega_{n}+a_{4}$. Here, $k_{p}=k$ for elastic behavior $(\mathrm{KEY}=0)$ and $k_{p}=0$ for plastic behavior (KEY = 1 or -1$)$.

(8) Compute the incremental displacement $\Delta \mu_{j}=\frac{\Delta \bar{F}_{j}}{\bar{K}_{j}}$

(9) Solve for the incremental quantity $\Delta \dot{\mu}_{j}=2 a_{1} \Delta \mu_{j}-a_{2} \dot{\mu}_{j}+a_{3} \Delta t \ddot{\mu}_{j}$

(10) Calculate the quantities $\mu_{j+1}=\mu_{j}+\Delta \mu_{j}$ and $\dot{\mu}_{j+1}=\dot{\mu}_{j}+\Delta \dot{\mu}_{j}$.

(11) Set the new value for the parameter KEY as follows

- When the system is behaving elastically at the beginning of the time step then the parameter $\mathrm{KEY}=0$ if $\mu_{y c}<\mu<\mu_{y t}, \mathrm{KEY}=1$ if $\mu>\mu_{y t}$ and $\mathrm{KEY}=-1$ if $\mu<\mu_{y c}$.

- When the system is behaving plastically in tension at the beginning of the time step then the parameter $\mathrm{KEY}=1$ if $\dot{\mu}>0$ and $\mathrm{KEY}=0$ if $\dot{\mu}<0$.

- When the system is behaving plastically in compression at the beginning of the time step then the parameter $\mathrm{KEY}=-1$ if $\dot{\mu}<0$ and KEY $=0$ if $\dot{\mu}>0$.

(12) Compute $\ddot{\mu}_{j+1}=-\frac{\omega_{n}^{2}}{a_{y}} \ddot{u}_{g j+1}-2 \zeta_{n} \omega_{n} \dot{\mu}_{j+1}-\omega_{n}^{2} \bar{f}_{s}\left(\mu_{j+1}, \dot{\mu}_{j+1}\right)$. Here, $\bar{f}_{s}$ $\left(\mu_{j+1}, \dot{\mu}_{j+1}\right)$ is given as, $1-\left(\mu_{j}-\mu_{j+1}\right)$ if $\mathrm{KEY}=0,1$ if $\mathrm{KEY}=1$ and -1 if $\mathrm{KEY}=-1$.

(13) Repeat steps 5 to 12 for all discrete points of time $\left(j=1,2, \ldots, N_{p}\right.$, and $N_{p}$ is the total number of discrete points of time).

(14) The optimal normalized inelastic response is computed as $\mu\left(t_{m}\right)=\max _{1 \leq j \leq N_{p}}$ 
$\mid \mu\left(t_{j} \mid\right)$ and the corresponding set of optimization variables $A_{i}, B_{i}, i=$ $1,2, \ldots, N_{f}, \alpha_{1}$ and $\alpha_{2}$ defines the critical earthquake signal (equations 5 and 6$)$ and the associated critical inelastic response $u(t)=u_{y} \mu(t)$.

\section{Numerical results and discussions}

To illustrate the formulation developed in the preceding section the determination of optimal earthquake excitations for an elastic-plastic one storey steel frame is demonstrated in this section. The frame structure has a width $L=9.14 \mathrm{~m}$, height $h=6.25 \mathrm{~m}$ and modulus of elasticity $E=210 \mathrm{Gpa}$. The structure carries a total load of $9.66 \times 10^{3} \mathrm{~N} / \mathrm{m}$ and columns are made of $W 8 \times 24$ steel section. A similar structure was considered by Wang et al. [23] within the context of seismic assessment of inelastic structures. Additionally, the frame structure is taken to be located at a site with firm soil conditions and is subjected to a uniform earthquake ground motion at both support points. For purpose of dynamic analysis, it is assumed that the girder is sufficiently rigid to prevent rotation and that columns are massless. Accordingly, the frame structure is modelled as an elastic-plastic single-degree-of-freedom system (see Figure 2). The initial stiffness of columns is computed to be $1.49 \times 10^{5} \mathrm{~N} / \mathrm{m}$ and the natural frequency of the elastic linear system is $\omega_{n}=0.65 \mathrm{~Hz}$. A modal viscous damping of 0.03 is considered. The yield strength of the spring force in tension and compression is taken as $10^{4}$ and $-10^{4} \mathrm{~N}$, respectively. This, in turn, leads to defining yield displacements in tension and compression as 0.0671 and $-0.0671 \mathrm{~m}$, respectively.

\subsection{Quantification of constraints}

A set of 20 earthquake ground motions $\left(N_{r}=20\right)$ is used to quantify the constraint limits $E, M_{1}, M_{2}, M_{3}, M_{4}(\omega), M_{5}(\omega), \alpha_{1 l}, \alpha_{1 u}, \alpha_{2 l}$ and $\alpha_{2 u}[25,26]$. Table 1 summarizes relevant information, such as, Richeter's magnitude, peak values of ground acceleration, velocity and displacement and energy for each record. The table contains, also, the envelope parameters $\alpha_{1}$ and $\alpha_{2}$ that reflect the transient nature of each acceleration (see equations (5) and (6)). These records include digitized information on ground acceleration, velocity and displacement and each record is reported to be measured on firm soil. Based on numerical analysis of these records the quantities of the constraints appearing in equations (13) were computed as $E=4.17 \mathrm{~m} / \mathrm{s}^{1.5}, M_{1}=4.63$, $\mathrm{m} / \mathrm{s}^{2}(0.47 \mathrm{~g}), M_{2}=0.60 \mathrm{~m} / \mathrm{s}$ and $M_{3}=0.15 \mathrm{~m}$. The upper and lower bounds on the Fourier spectra $M_{4}(\omega)$ and $M_{5}(\omega)$ are shown in figure 3(a). It may be noted that $N_{r}=20$ was seen to produce considerably smooth upper and lower bounds on the Fourier coefficients of $\ddot{u}_{g}(t)$. The average dominant frequency 
of the ground accelerations is seen to be around $1.64 \mathrm{~Hz}$. The bounds on the envelope function parameters were determined to be $\alpha_{1 l}=0.07, \alpha_{1 u}=0.19$, $\alpha_{2 l}=0.59$ and $\alpha_{2 u}=0.67$. The envelope functions corresponding to these values are illustrated in figure $3(\mathrm{~b})$. These values indicate that the positivity constraint of the envelope parameters, and, the constraint $\alpha_{2}>\alpha_{1}$ (see equation 6) are implicitly satisfied. The parameter $\beta$ of the numerical integration algorithm is selected as 0.25 . The time step $\Delta t$ is taken as $0.005 \mathrm{sec}$ which was found to give satisfactory results in the numerical integration of the equation of motion. Additionally, the convergence limits $\ell_{1}$ and $\ell_{2}$ were taken as $10^{-4}$ and $10^{-6}$, respectively. The frequency content for $\ddot{u}_{g}(t)$ is taken as $(0-25)$ Hz. The resulting constrained nonlinear optimization problem is tackled by using the sequential quadratic optimization algorithm 'fmincon' of the Matlab optimization toolbox [24]. As mentioned earlier, this algorithm requires the specification of an initial guess for the vector of optimization variables $x$. In the numerical calculations, alternative initial starting solutions, within the visible region, were examined and it was found that all these guesses lead to the same optimal solution. Furthermore, in distributing $\omega_{i}, i=1,2, \ldots, N_{f}$ in the interval $\left(\omega_{0}, \omega_{c}\right)$, (see equation 5 ) it was found advantageous to select one of these $\omega_{i}$ to coincide exactly with the structure natural frequency and also to place relatively more points within the modal half-power bandwidth. To select the number of frequency terms $N_{f}$ a parametric study was carried out and $N_{f}=50$ was found to give satisfactory results. Accordingly, the total number of the optimization variables were 52 .

\subsection{Dissipated energy}

To gain more insights into the nature of optimal earthquake loads computed it is of interest to quantify various forms of energy dissipated by the inelastic system. Several authors employed the energy dissipated by the structure in characterizing response analysis of structures to dynamic loading [12,27-29]. These energy terms can be quantified by integrating the structure equation of motion. Thus, the energy balance for the inelastic system can be written as (see equation (2))

$$
\int_{0}^{u} m \ddot{u}(t) d u+\int_{0}^{u} c \dot{u}(t) d u+\int_{0}^{u} f_{s}(u, \dot{u}) d u=-\int_{0}^{u} m \ddot{u}_{\mathrm{g}}(t) d u
$$

The right side of the above equation represents the input energy to the structure since ground starts shaking until it comes to rest. The first energy term of the left side is the kinetic energy $E_{K}(t)$ of the mass associated with its motion 
relative to the ground and is given as

$$
E_{K}(t)=\int_{0}^{u} m \ddot{u}(t) d u=\int_{0}^{\dot{u}} m \ddot{u}(t) d \dot{u}=\frac{m[\dot{u}(t)]^{2}}{2}
$$

The second term of the left side of equation (17) represents the energy dissipated by viscous damping $E_{D}(t)$ given by

$$
E_{D}(t)=\int_{0}^{u} c \dot{u}(t) d u=\int_{0}^{t} c[\dot{u}(t)]^{2} d t
$$

The third term of equation (17) is the sum of the recoverable strain energy $E_{S}(t)$ and the energy dissipated by yielding $E_{Y}(t)$ and are given as

$$
\begin{array}{r}
E_{S}(t)=\frac{\left[f_{s}(t)\right]^{2}}{2 k} \\
E_{Y}(t)=\int_{0}^{u} f_{s}(u, \dot{u}) d u-E_{S}(t)=\int_{0}^{t} \dot{u}(t) f_{s}(u, \dot{u}) d t-E_{S}(t)
\end{array}
$$

The parameter $k$ appearing in the above equation, is the initial stiffness of the inelastic system. In the present study, the time-variation of energy terms given in equations (18-20) are employed in quantifying and characterizing various forms of energy dissipated by the inelastic system.

\subsection{Results and discussions}

Alternative constraint scenarios considered in deriving critical seismic inputs are listed in table 2. The numerical results obtained for the elastic-plastic structure are presented in figures (4) to (8) and table 3. The critical earthquake load computed for constraint scenario 1 (see table 2) is presented in figure (4). This figure depicts the time history of the ground acceleration (figure 4(a)) and also the Fourier amplitude spectra of $\ddot{u}_{g}(t)$ (figure 4(b)). Similar results for constraint scenario 4 are presented in figure (5). The critical envelope function and the convergence of the objective function, for case 4 , are provided in figures $6(\mathrm{a})$ and $6(\mathrm{~b})$, respectively. The associated structure inelastic response is plotted in figure 7(a), and, the hysteresis loops for the restoring force-displacement of the system is shown in figure $7(\mathrm{~b})$. With an aim to investigate the influence of the structure yield strength on the computed critical earthquake accelerations and the associated inelastic response a parametric study was carried out. The yield strength was varied while other 
parameters are kept unchanged and the earthquake acceleration is computed for each value of yield strength by re-solving the same optimization problem. Figure (8) shows part of these results. A similar study to investigate the effect of the variation of the damping ratio on the structure response was also carried out. The results of this parametric study is presented in table 4 . The time-variation of different energy forms dissipated by the inelastic system are provided in figure $(9(\mathrm{a}))$. To understand the influence of modelling the structure inelastic behavior on critical earthquake loads, critical $\ddot{u}_{g}(t)$ for the elastic linear structure were also determined. Some of these results are provided in figures (9-11). Based on extensive study of the numerical results, the following observations are made

(1) It is observed that the magnitude of critical inelastic response produced and frequency content of critical excitations are strongly dependent on constraints imposed (see figures 4 and 5 and table 3). If available knowledge on future earthquake is limited to energy and PGA (case 1), the critical excitation is highly resonant and response produced is overly conservative (see figure 4(b) and table 3). Additional constraints on bounds on Fourier amplitude spectra (case 4) makes the critical excitations realistic in terms of their frequency content and responses that they produce. To see this, the critical response produced by alternative constraint scenarios can be compared with the highest inelastic displacement of 0.10565 $\mathrm{m}$ that is produced by the recorded motions $\left\{\ddot{v}_{g n}(t)\right\}_{n=1}^{N_{r}}$. Thus for the case of constraints on energy and PGA (case 1), the critical response is 4.17 times the highest response produced by past records while, for case 4 this ratio reduces to 1.82 . It is also observed that the critical ground acceleration for the inelastic structure possesses a peak amplitude at a frequency close to the natural frequency of the elastic system (see figures $4(\mathrm{~b})$ and 5(b)). This peak, however, is seen to be significantly smaller than that observed for the case of the elastic structure (figure 10(b)). Notwithstanding this, it is observed that the dominant frequency of the ground acceleration is close to the average dominant frequency of past record ground motions (see figure $5(\mathrm{~b})$ ).

(2) The convergence rate of the objective function with respect to the number of iterations is seen to be faster for the elastic structure compared to that of the inelastic structure. Thus, for case 4 , the objective function for the linear case reaches initial convergence to the optimal solution within about 1500 iterations, the corresponding number of iterations when inelastic behavior is considered is more than 8000. The final convergence of the objective function for the elastic system is achieved within about 3500 iterations, while in the case inelastic system, the final convergence is achieved within around 15000 iterations (see figures 6(b) and 11(b)). It was, also, observed that the CPU time necessary for the convergence of the objective function in the case of the inelastic system is around four times that for the elastic system. 
(3) It is evident from the numerical results on critical ductility factor $\mu(t)$ and associated displacement response for the inelastic structure that the time variation of the structure deformation differs from that of the elastic system (see figures $7(\mathrm{a})$ and 11(a)). Unlike the elastic system, the inelastic system after it has yielded does not oscillate about its initial equilibrium position. Yielding causes the structure to drift from its initial equilibrium position and system oscillates around a new equilibrium position until this gets shifted by another yielding. Accordingly, after the ground stops shaking, the structure comes to rest at a position different from its initial equilibrium position. In other words, the structure permanent deformation remains after ground stops shaking. For instance, the permanent displacement response of the structure, for case 4, was seen to be $0.0533 \mathrm{~m}$. Additionally, the maximum value of the structure deformation and the point at which it occurs are different for the inelastic system compared to those of the elastic system. Thus, the peak response for the inelastic structure is around $0.1922 \mathrm{~m}$ while the corresponding value for the elastic system was $0.4705 \mathrm{~m}$. These peaks occur at $t=6.58$ sec and $t=7.96 \mathrm{sec}$ for the inelastic and elastic systems, respectively. The maximum value of the ductility factor was computed to be about 2.86 .

(4) The influence of the structure yield strength on the computed earthquake acceleration is seen to be significant. Thus, the decrease in the structure yield strength, $f_{y}$, is seen to spread the amplitude of the fourier spectra of the critical earthquake acceleration across higher frequencies. This was observed to be compensated with a decrease in the peak of the Fourier amplitude of $\ddot{u}_{g}(t)$ at the structure natural frequency. Furthermore, it is also observed that for lower yield values the structure yields more frequently and for longer intervals (see figure 8(a)). The structure dissipated energy, due to yielding, is seen to increase for higher yield strength (see figure 8(b)). Additionally, with higher yielding strength, the structure ductility factor is seen to reduce. Thus, for case 4 , the ductility factor associated with the yielding limits $10^{4}, 2 \times 10^{4}$ and $3 \times 10^{4} \mathrm{~N}$ were 2.86, 2.32 and 2.11 , respectively. The associated peak responses were $0.1922,0.2885$ and $0.4216 \mathrm{~m}$, respectively. The corresponding permanent deformation of the structure after ground stops shaking are 0.0533, 0.0741 and 0.0874 $\mathrm{m}$, respectively.

(5) The input energy to the inelastic system due to the earthquake acceleration $\ddot{u}_{g}(t)$ is, primarily, dissipated by yielding and damping of the structure(see figure 9(a)). Unlike the elastic system, the kinetic and recoverable strain energy terms for the inelastic system are small and diminish near the end of the ground shaking (figure 9). The energy dissipated by yielding is significantly higher than that dissipated by damping. Furthermore, viscous damping dissipates less energy from the inelastic system compared to that for the elastic system. This is not surprising given that velocity response is higher for the elastic system. It is also obvious that 
input energy to the inelastic system differs from the energy input to the linear elastic system. The repeated yielding of the elastic-plastic system indicates the level of the structure damage and the associated permanent deformation caused to it.

(6) The influence of the variation of the damping ratio on the structure inelastic deformation was seen to be significant. As might be expected with increasing the damping ratio, the structure maximum inelastic response is seen to reduce (see table 4 ). Thus, for case 4 , the structure maximum inelastic deformation was computed to be $0.1922 \mathrm{~m}$ for $\zeta=0.03$. This value reduces to 0.1624 if $\zeta=0.05$. The ductility factor reduces from 2.86 to 2.42 due to increase of damping ratio from 0.03 to 0.05 . Furthermore, it was also seen that the permanent deformation of the inelastic system reduces for higher values of damping ratio (see table 4).

In order to study the sensitivity of critical response with respect to variations in the constraint quantities $E, M_{1}, M_{2}, M_{3}, M_{4}(\omega), M_{5}(\omega), \alpha_{1 l}, \alpha_{1 u}, \alpha_{2 l}$ and $\alpha_{2 u}$, a sensitivity analysis using numerical methods is carried out. To study the sensitivity of critical response with respect to a specific parameter, the value of this parameter is changed by $1 \%$ while other parameters are held fixed at their respective specified values. The optimization problem is re-solved with this change in place. This leads to the calculation of the percentage change in the critical response, denoted by $\epsilon_{1}$, and also the ratio of change in the response value to the change in the parameter value, denoted by $\epsilon_{2}$. Table 5 summarizes the results of this calculation for constraint scenario 4 . It can be observed from this table that changes in the energy constraint and bounds on the fourier spectra alter the optimum solution considerably compared to similar changes in other parameters. The optimum solution is less sensitive to constraints on peak values of acceleration, velocity and displacement and bounds on the envelope parameters.

Finally, with a view to investigate the effect of the natural frequency of the structure on the derived critical ground acceleration and associated structure inelastic response, an additional study is carried out. Herein, the structure natural frequency was varied (by varying the structure mass while stiffness is kept unchanged) and the critical acceleration is computed. These results are presented in figure (12). It follows from this figure that the natural frequency of the linear system significantly influences the computed critical $\ddot{u}_{g}(t)$ and the associated structure inelastic response. If the structure fundamental natural frequency is close to the dominant frequency of the site under consideration high inelastic deformation is produced. 


\section{Conclusions}

The modelling of earthquake ground motion as design inputs for inelastic single-degree-of-freedom structures is studied. The earthquake acceleration is expanded in terms of a Fourier series, of unknown coefficients, that is modulated by an enveloping function. The coefficients of the series representation and the envelope parameters are computed such that the structure inelastic response normalized to yield displacement is maximized under a set of predefined constraints. These constraints are taken to reflect known characteristics of actual recorded ground motions at the site under consideration. Particularly, constraints on the total energy of the earthquake signal, upper and lower bounds on the Fourier coefficients of the ground acceleration are considered. The constraints, also, contain upper limits on PGA, PGV and PGD and mathematical bounds on the envelope parameters. The structure force-displacement relation is taken to possess an elastic-plastic behavior. The resulting nonlinear optimization problem is solved by using the sequential quadratic optimization method. Several aspects which are relevant to the problem are investigated. It is shown that critical earthquake loads for the inelastic structure differ from that computed for the same structure with linear structural behavior. Similarly, the time variation of the structure deformation differs from that of the elastic system. Unlike the elastic system, the inelastic system after it has yielded does not oscillate about its initial equilibrium position. Yielding causes the structure to drift from its initial equilibrium position and system oscillates around a new equilibrium position until this gets shifted by another yielding. The present study, also, examined influences of the variations of the structure yield strength and damping ratio on the computed earthquake load and associated structure response. It was found that for lower yield values the structure yields more frequently and for longer intervals. Additionally, with higher yielding strength, the structure maximum response increases. The influence of damping ratio was seen to be significant in reducing the structure inelastic deformation. It is, also, shown that the inelastic structure dissipates the input energy, mainly, through yielding and damping. Furthermore, the time-variation of alternative energy forms for the inelastic structure differ from those for the linear structure.

The proposed formulation was demonstrated with reference to seismic inelastic response analysis of a simple frame structure. Given the complexity of engineering structures, it is thus of significant interest to extend this formulation to multi-degree-of-freedom structures. This can be achieved by integrating tools of nonlinear finite element analysis and nonlinear optimization techniques. It is also of significance to investigate effects of treating nonlinear damping models in computing critical earthquake inputs for inelastic structures. A natural development of the method of critical earthquake excitations, presented in this work, is the seismic-resistant design of inelastic structures subjected to 
system-dependent critical loads. Herein, information on the seismic load and, also, on the structure parameters would be specified partially. 


\section{References}

[1] J.J. Bommer, A.B. Acevedo, "The use of real earthquake accelerograms as input to dynamic analysis." Journal of Earthquake Engineering, 1 (2004) 43-91.

[2] R.F. Drenick, "Model-free design of aseismic structures." Journal of Engineering Mechanics, 96 (1970) 483-493.

[3] M. Shinozuka, "Maximum structural response to seismic excitations." Journal of Engineering Mechanics, 96 (1970) 729-738.

[4] R.N. Iyengar, "Matched inputs." Report 47, Series J., Center of Applied Stochastics, Purude University, West Lafayette, Ind. (1970).

[5] I. Takewaki, "Seismic critical excitation method for robust design: A review." Journal of Structural Engineering, 128 (2002) 665-672.

[6] A.M. Abbas, C.S. Manohar, "Investigations into critical earthquake load models within deterministic and probabilistic frameworks". Earthquake Engineering and Structural Dynamics, 31 (2002) 813-832.

[7] A.M. Abbas, Deterministic/Reliability-based critical earthquake load models for linear/nonlinear engineering structures, Ph. D. thesis, Department of Civil Engineering, Indian Institute of Science, 2002.

[8] R.N. Iyengar, C.S. Manohar, "Nonstationary random critical excitations." Journal of Engineering Mechanics, 133 (1987) 529-541.

[9] R.N. Iyengar, "Worst inputs and a bound on the highest peak statistics of a class of non-linear systems." Journal of Sound and Vibration, 25 (1972) 29-37.

[10] R.F. Drenick, "The critical excitation of nonlinear systems." Journal of Applied Mechanics, 18 (1977) 333-336.

[11] A.J. Philippacopoulos, P.C. Wang, "Seismic inputs for nonlinear structures." Journal of Engineering Mechanics, 110 (1984) 828-836.

[12] B.D. Westermo, "The critical excitation and response of simple dynamic systems." Journal of Sound and Vibration, 100 (1985) 233-242.

[13] A.A. Pirasteh, J.L. Cherry, J.A. Balling, "The use of optimization to construct critical accelerograms for given structures and sites." Earthquake Engineering and Structural Dynamics, 16 (1988) 597-613.

[14] I. Takewaki, "Probabilistic critical excitation for MDOF elastic-plastic structures on compliant ground." Earthquake Engineering and Structural Dynamics, 30 (2001) 1345-1360.

[15] I. Takewaki, "Critical excitation for elastic-plastic structures via equivalent linearization." Probabilistic Engineering Mechanics, 17 (2002b) 73-84. 
[16] A.M. Abbas, C.S. Manohar, "Reliability-based critical earthquake load models. Part 2: Nonlinear structures." Journal of Sound and Vibration, 287 (2005) 883900 .

[17] M. Shinozuka, Y. Sato, "Simulation of nonstationary random process." Journal of Engineering Mechanics, 91 (1967) 11-40.

[18] M. Shinozuka, L. Henry, "Random vibration of a beam column." Journal of Engineering Mechanics, 91 (1965) 123-143.

[19] A. Baratta, I Elishakoff, G Zuccaro, M. Shinozuka, "A generalization of the Drenick-Shinozuka model for bounds on the seismic response of a singledegree-of-freedom system." Earthquake Engineering and Structural Dynamics, 27 (1998) 423-437.

[20] R.F. Drenick, "Aseismic design by way of critical excitation." Journal of Engineering Mechanics, 99 (1973) 649-667.

[21] P.C. Wang, C.B. Yun, "Site-dependent critical design spectra." Earthquake Engineering and Structural Dynamics, 7 (1979) 569-578.

[22] J.S. Arora, Introduction to optimum design, Elsevier Academic Press, San Diego, 2004.

[23] J. Wang, L. Fan, S. Qian, J. Zhou, "Simulation of non-stationary frequency content and its importance to seismic assessment of structures." Earthquake Engineering and Structural Dynamics, 31 (2002) 993-1005.

[24] T. Caleman, M.A. Branch, and A. Grace, Optimization toolbox for the use with Matlab, user's guide version 2(Release 11), The MATH WORKS Inc., USA, 1999.

[25] COSMOS, Consortium organizations for strong-motion observation systems, "http://db.cosmos-eq.org/scripts/default.plx", 2005.

[26] A.F. Shakal, M.J. Huang, Standard tape format for CSMIP strong-motion data tapes, California strong motion instrumentation program, Report OSMS 85-03, 1985 .

[27] I. Takewaki, "Bound of earthquake input energy." Journal of Structural Engineering, 130 (2004) 1289-1297.

[28] C.M. Uang, V.V. Bereto, "Evaluation of seismic energy in structures." Earthquake Engineering and Structural Dynamics, 19 (1990) 77-90.

[29] T.F. Zahrah, W.J. Hall, "Earthquake energy absorbtion in sdof structures." Journal of Structural Engineering, 110 (1984) 1757-1772. 

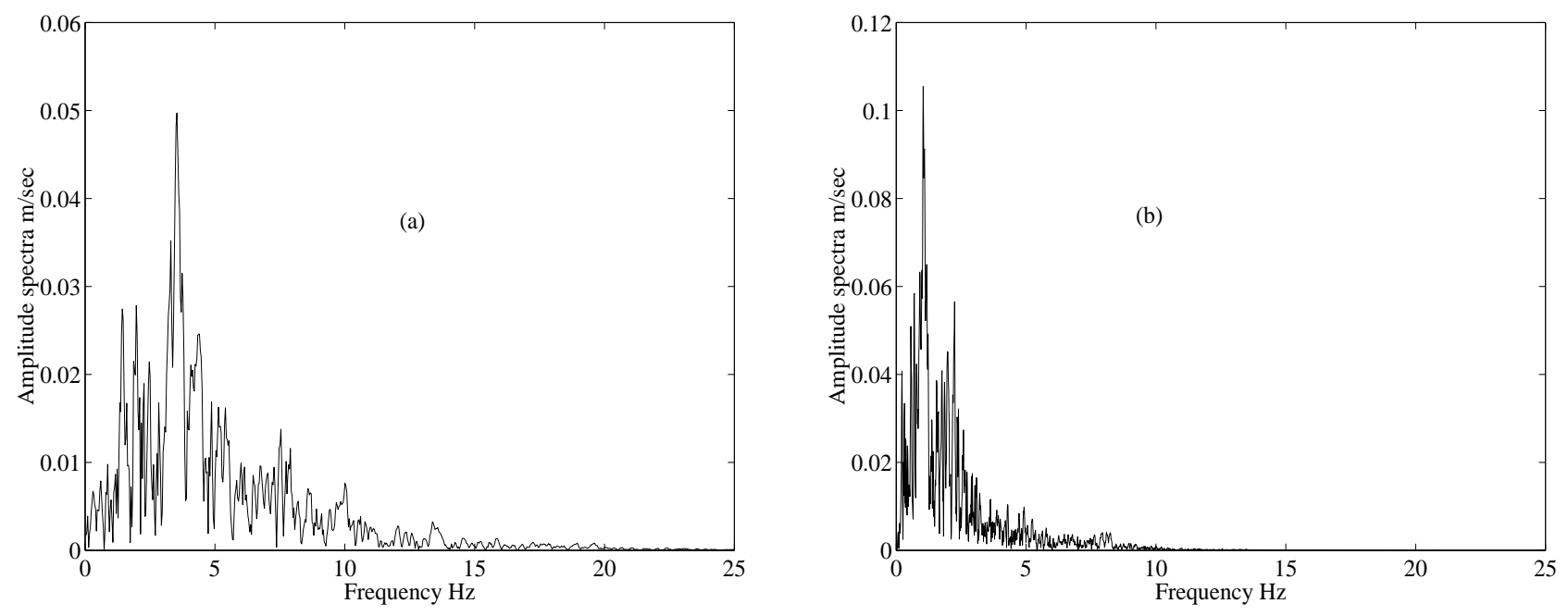

Fig. 1. Fourier amplitude spectra for recorded ground accelerations (a) San Fernando 1971, N69W (b) Hyogoken-Nanbu 1995, NIS0 $(25,26)$

(a)

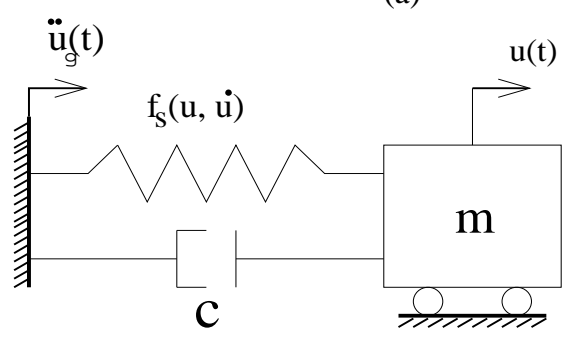

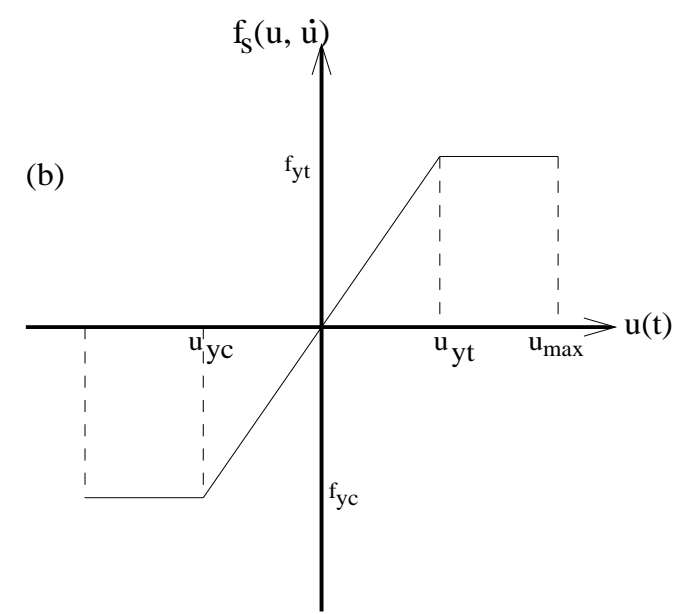

Fig. 2. (a) Inelastic single-degree-of-freedom system (b) Elastic-plastic behavior 

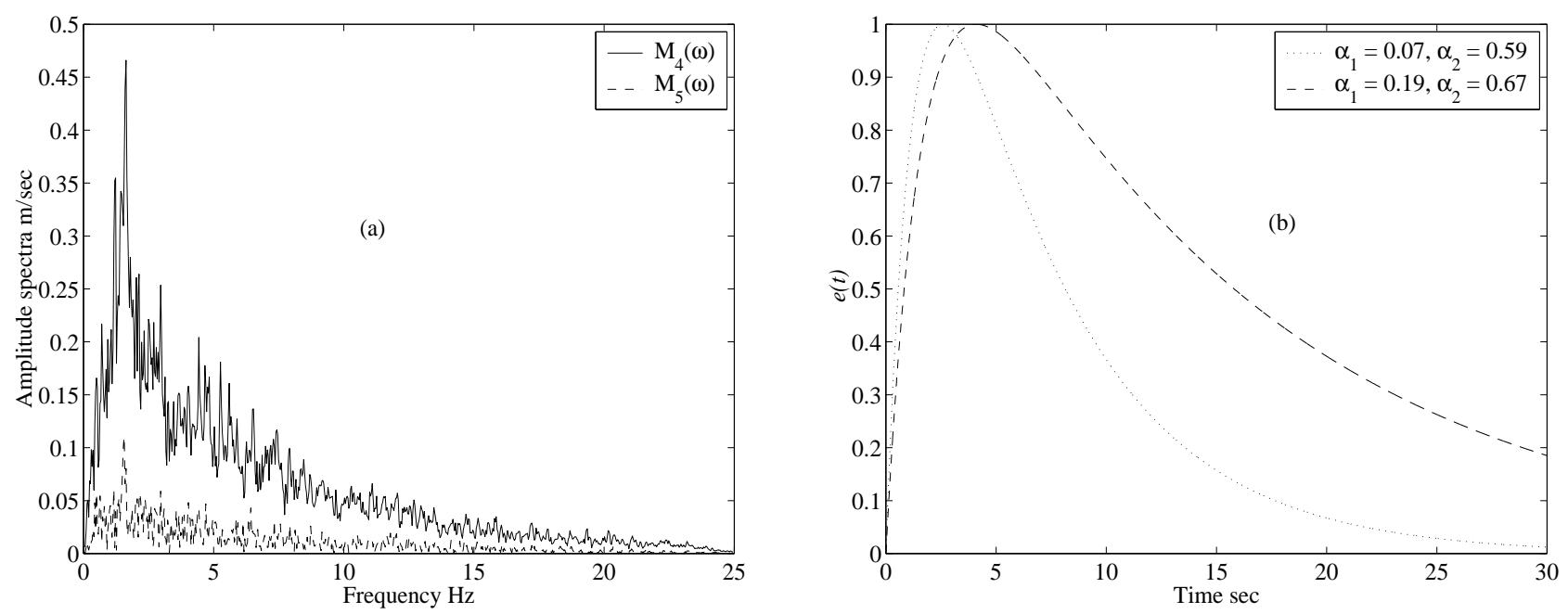

Fig. 3. Constraint bounds on ground acceleration (a) Upper and lower Fourier bounds (b) Envelope function
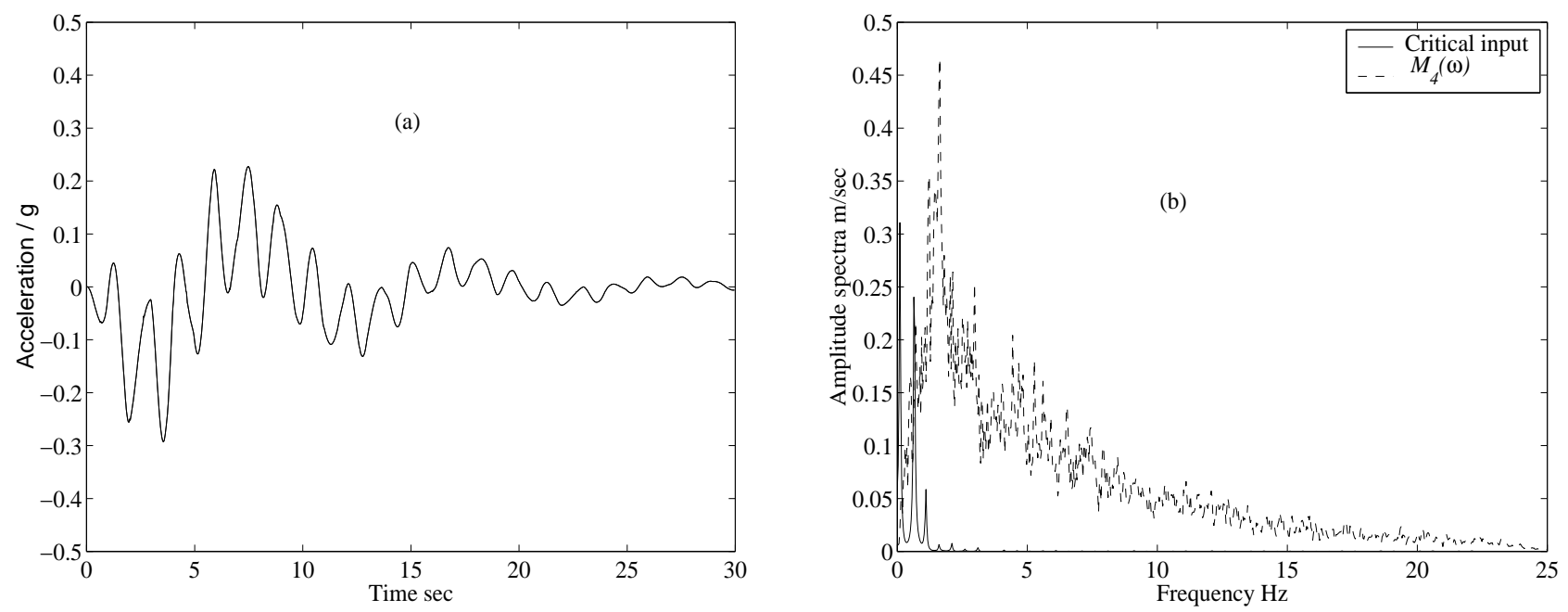

Fig. 4. Critical acceleration $\ddot{u}_{g}(t)$ for inelastic system; case (1) (a) Time history (b) Fourier amplitude spectra 

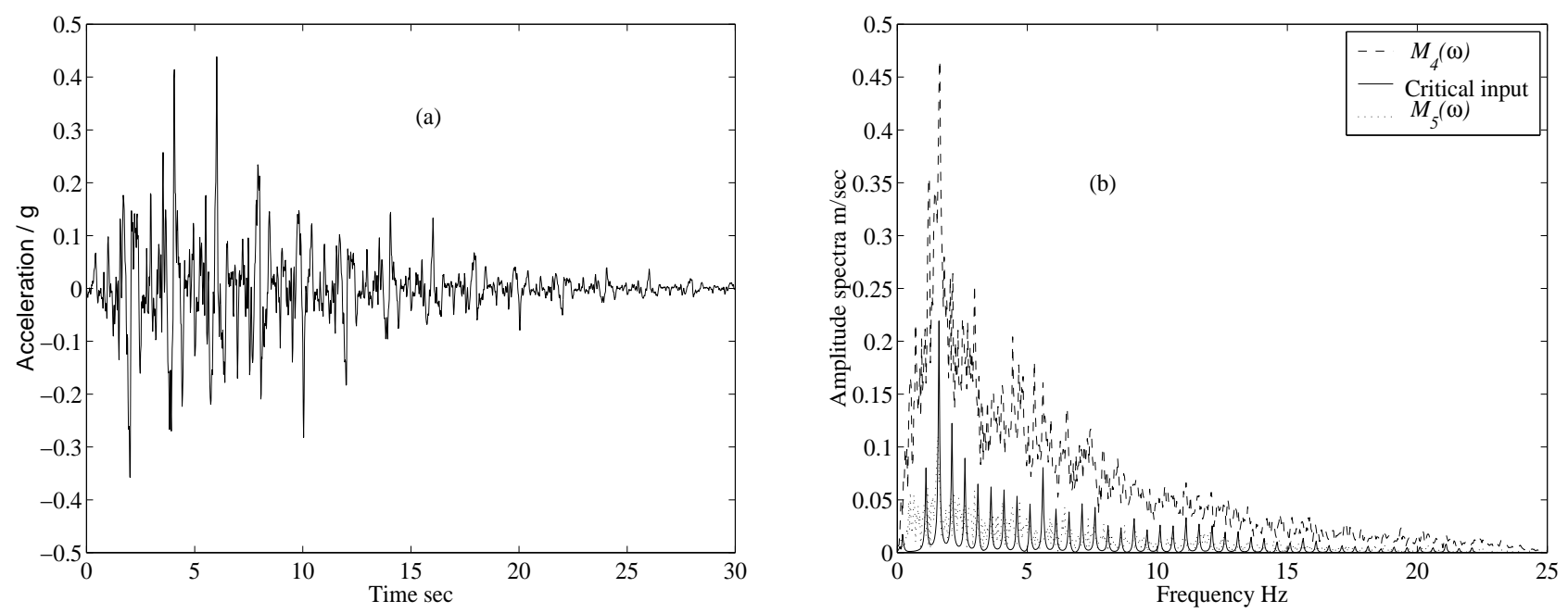

Fig. 5. Critical acceleration $\ddot{u}_{g}(t)$ for inelastic system; case (4) (a) Time history (b) Fourier amplitude spectra
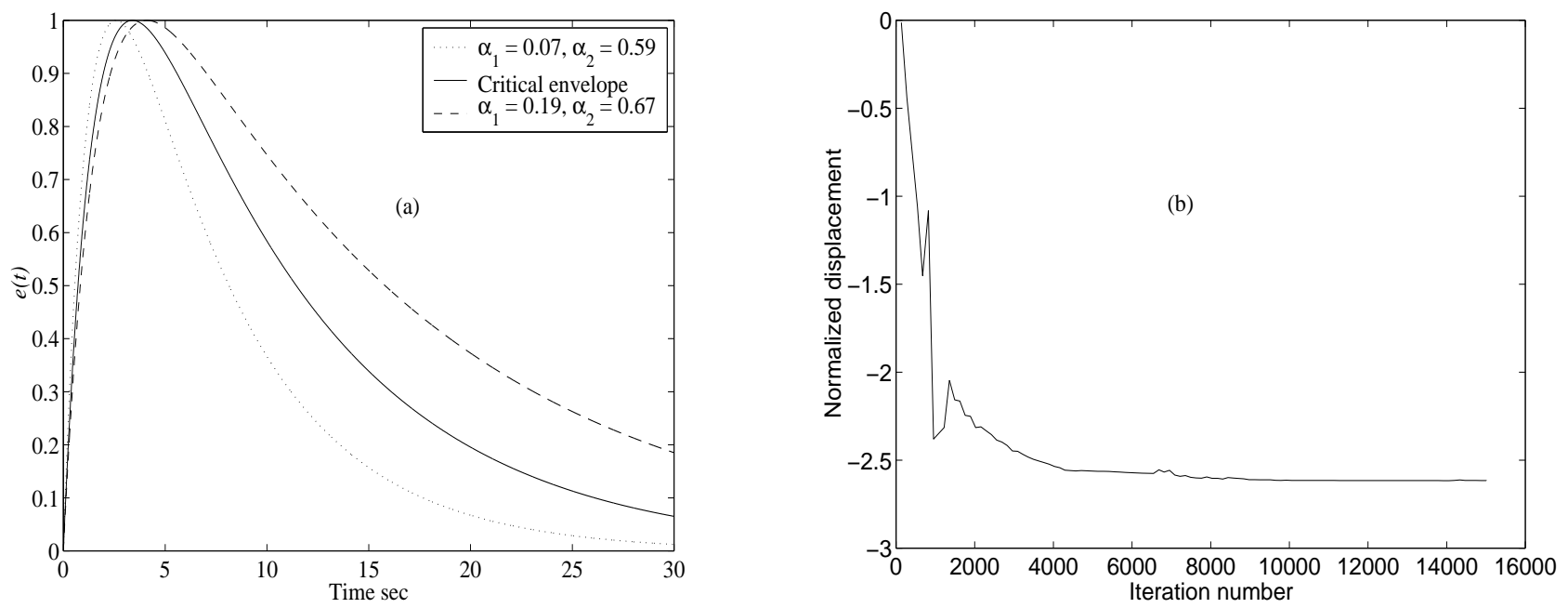

Fig. 6. Critical envelope function and convergence of objective function; case (4) (a) Envelope function (b) Convergence of objective function 

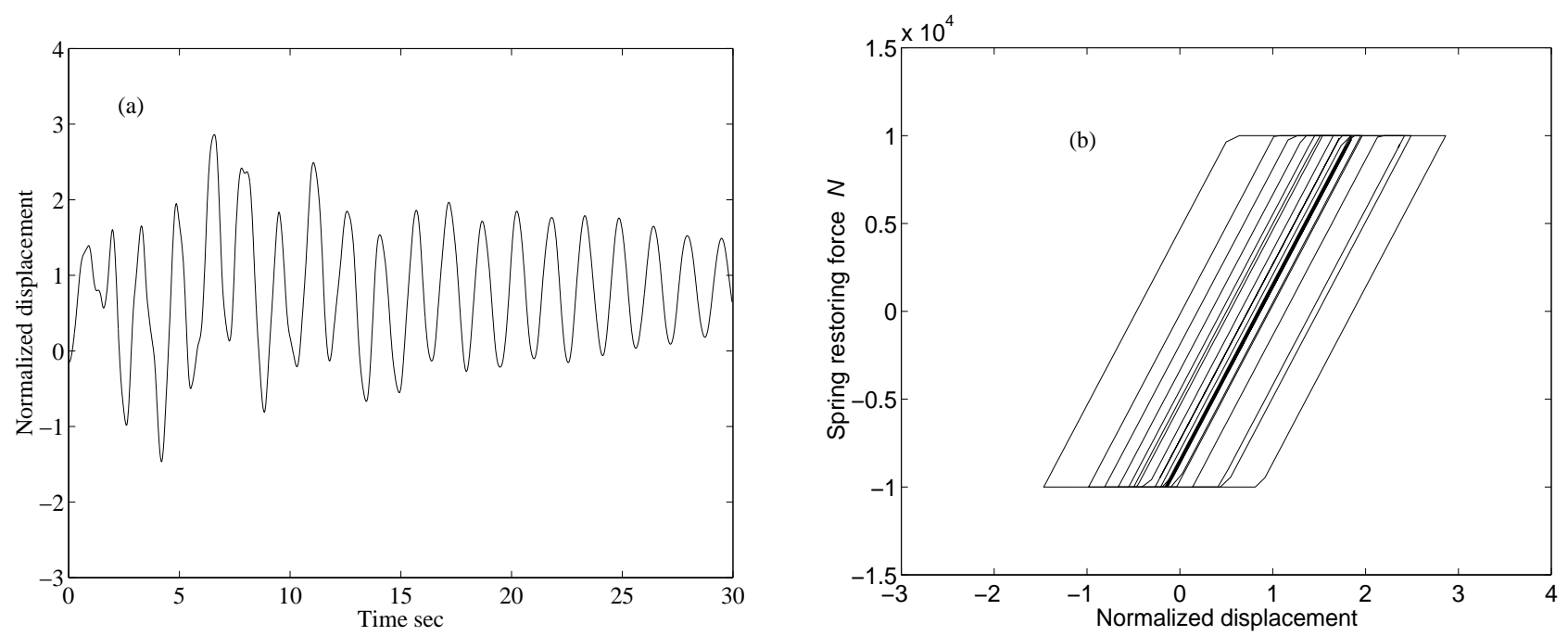

Fig. 7. Response of inelastic system; case (4) (a) Inelastic normalized displacement (b) Restoring force-displacement hysteretic loops
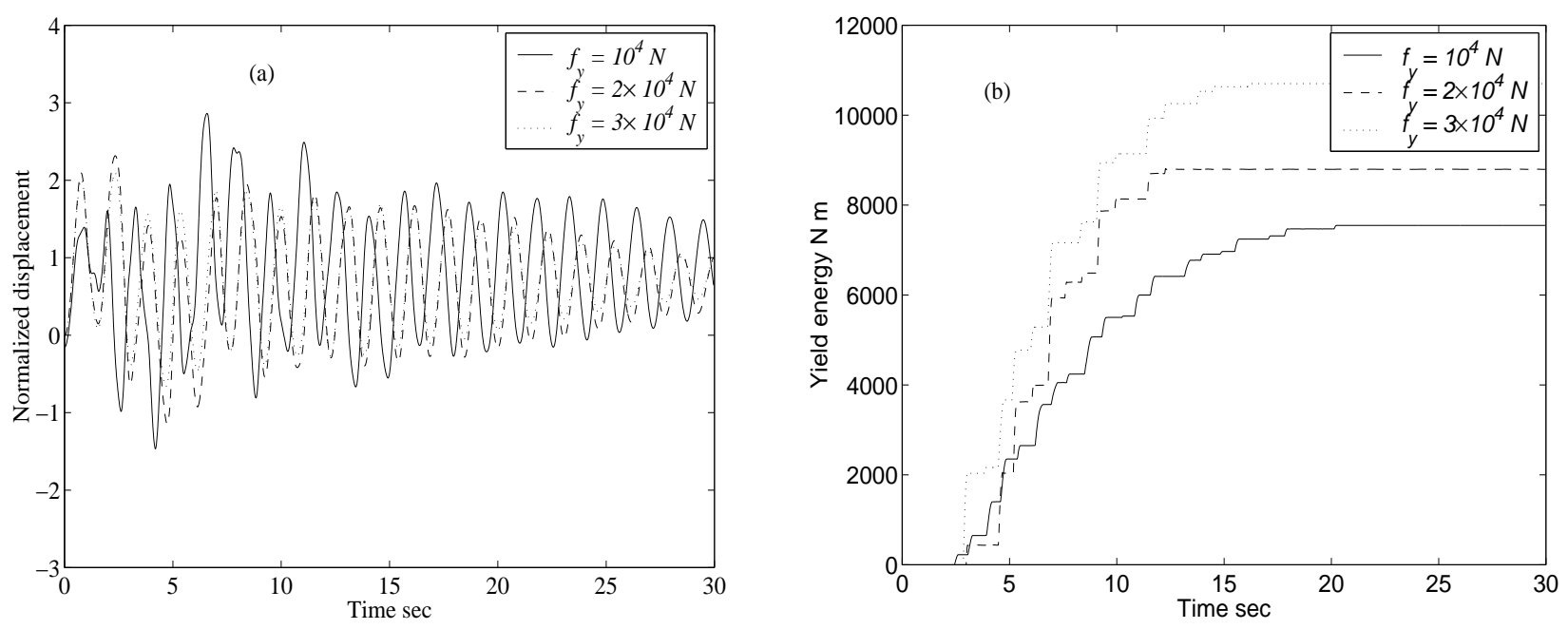

Fig. 8. Influence of yield strength on inelastic system; case (4) (a) Normalized response (b) Energy dissipated by yielding 

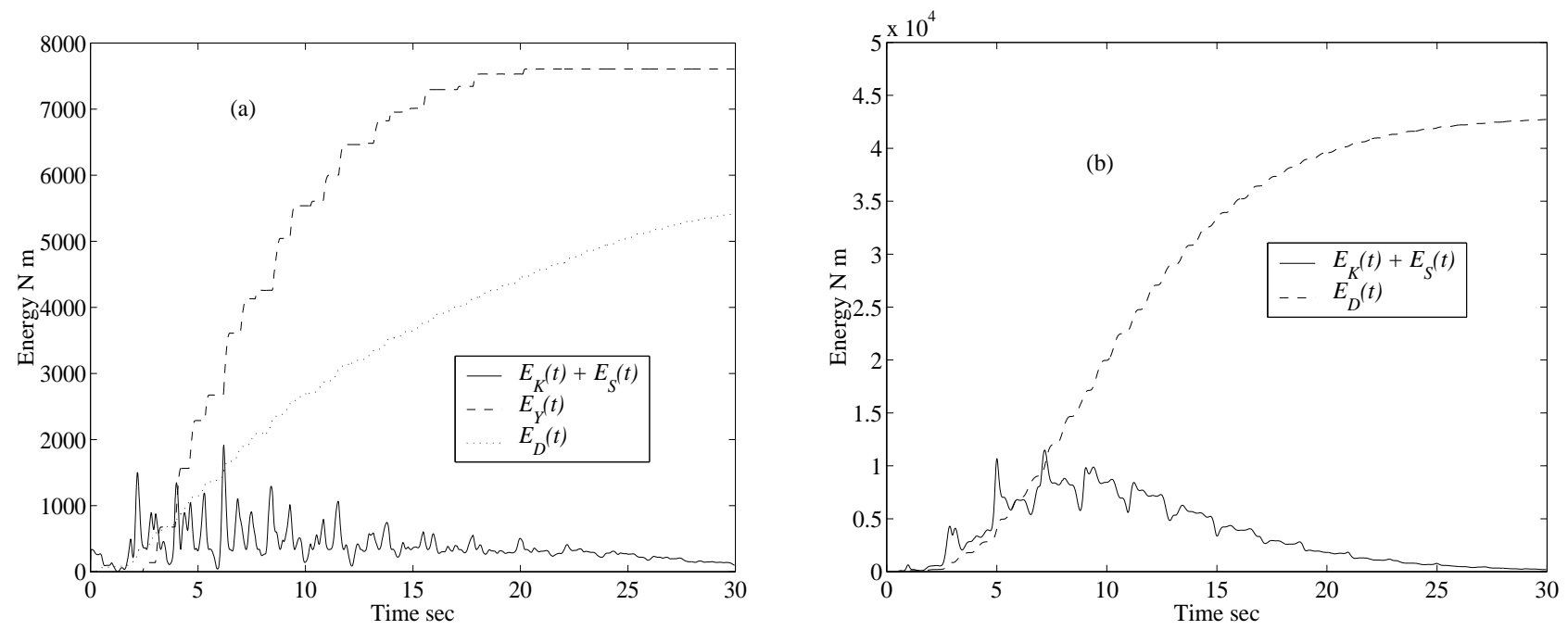

Fig. 9. Time-variation of dissipated energy; case (4) (a) Elastic-plastic system (b) Linear elastic system
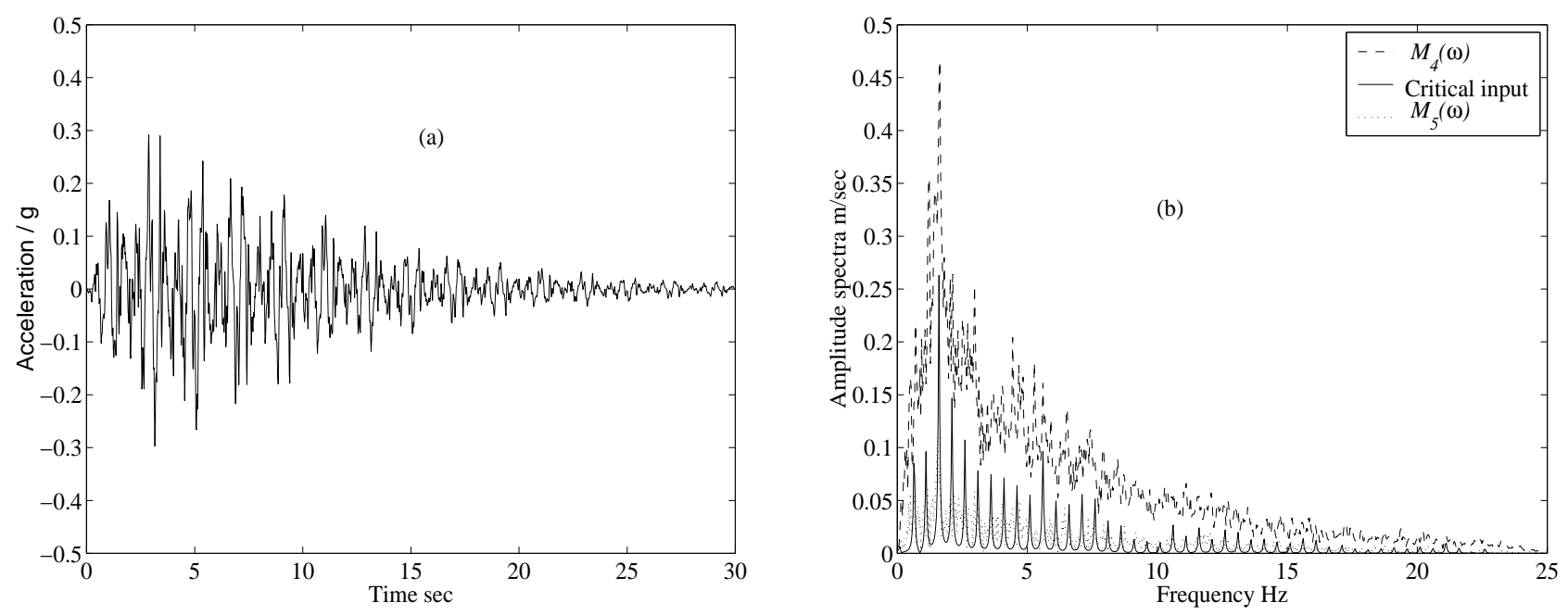

Fig. 10. Critical acceleration $\ddot{u}_{g}(t)$ for linear system; case (4) (a) Time history (b) Fourier amplitude spectra 

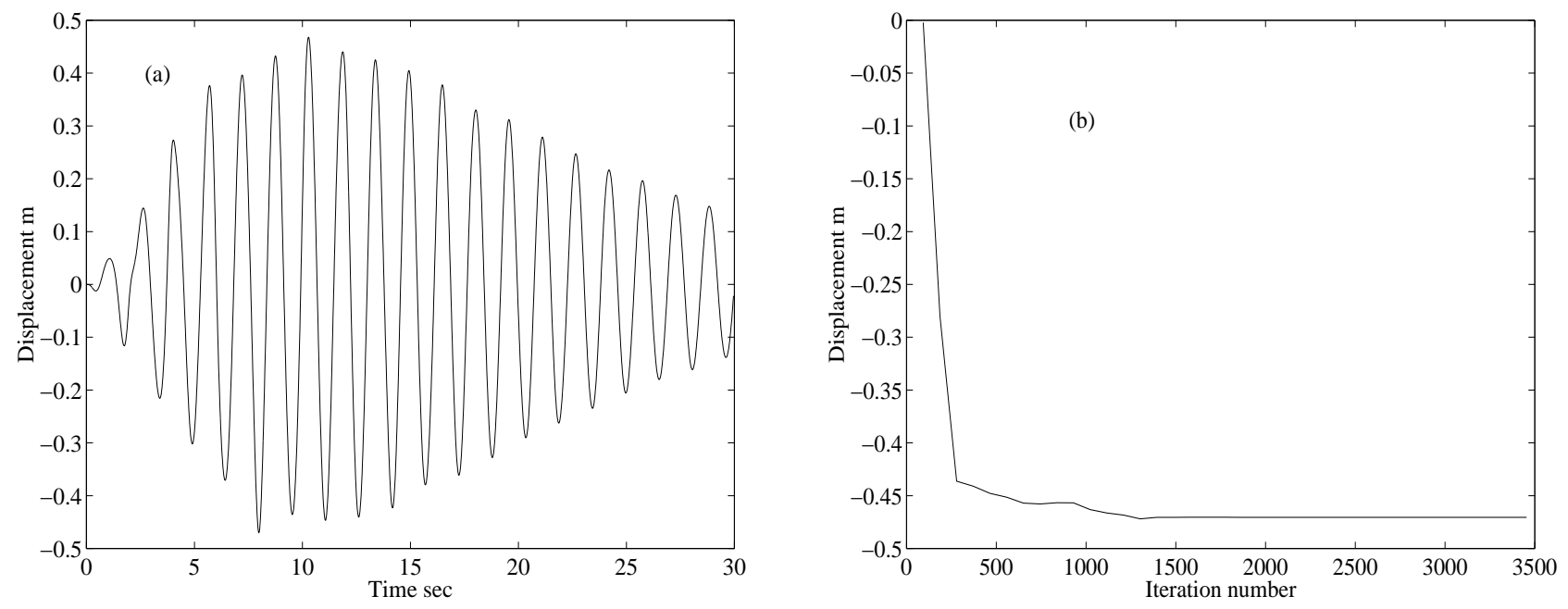

Fig. 11. Elastic system; case (4) (a) Critical displacement response (b) Convergence of objective function

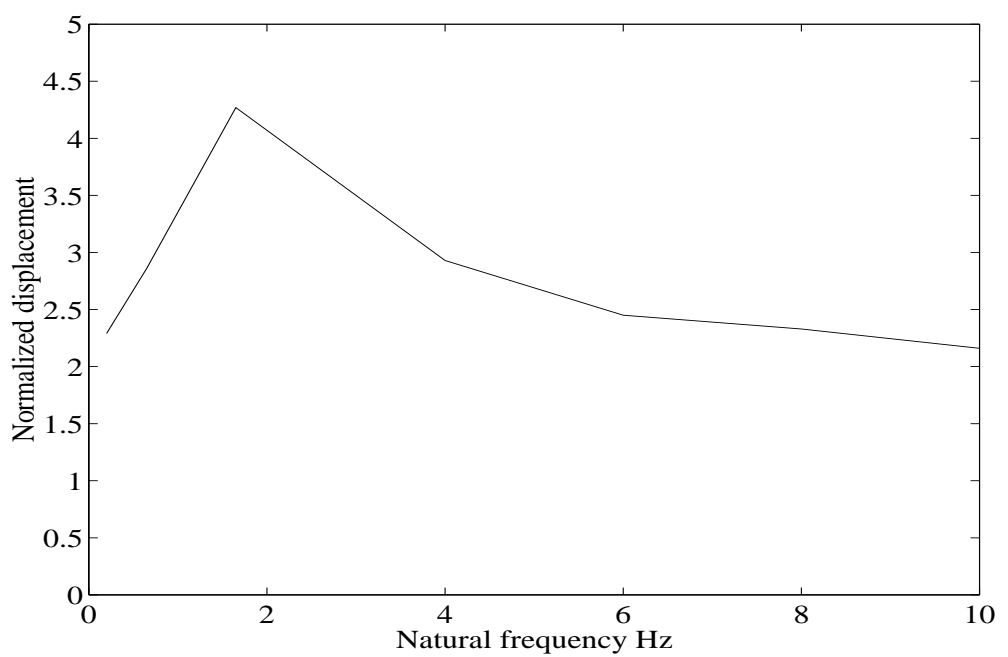

Fig. 12. Normalized inelastic response versus natural frequency; case (4) 
Table 1

Information on past recorded ground motions for a firm soil site $[27,28]$

\begin{tabular}{|c|c|c|c|c|c|c|c|c|c|c|}
\hline Earthquake & $\begin{array}{l}\text { Magn- } \\
\text { itude }\end{array}$ & $\begin{array}{l}\text { Epicen- } \\
\operatorname{ter}(\mathrm{km})\end{array}$ & $\begin{array}{l}\text { Comp- } \\
\text { onent }\end{array}$ & $\begin{array}{l}\text { PGA } \\
\left(\mathrm{m} / \mathrm{s}^{2}\right)\end{array}$ & $\begin{array}{l}\mathrm{PGV} \\
(\mathrm{m} / \mathrm{s})\end{array}$ & $\begin{array}{l}\text { PGD } \\
(\mathrm{m})\end{array}$ & $\begin{array}{l}\text { Energy } \\
\left(\mathrm{m} / \mathrm{s}^{1.5}\right)\end{array}$ & $\alpha_{1}$ & $\alpha_{2}$ & Site \\
\hline Mamoth lakes & & & $\mathrm{W}$ & 4.02 & 0.21 & 0.05 & 3.73 & 0.07 & 0.48 & Convi \\
\hline 05.25 .1980 & 6.2 & 1.5 & S & 3.92 & 0.23 & 0.05 & 4.01 & 0.11 & 0.36 & Gree \\
\hline Loma prieta & & & W & 3.91 & 0.31 & 0.07 & 3.82 & 0.09 & 0.32 & \\
\hline 10.18.1989 & 7.0 & 9.7 & S & 4.63 & 0.36 & 0.11 & 2.61 & 0.08 & 0.45 & Capitc \\
\hline Morgan hill & & & $\mathrm{S} 60 \mathrm{E}$ & 3.06 & 0.40 & 0.07 & 2.33 & 0.12 & 0.17 & Halls \\
\hline 04.24 .1984 & 6.1 & 4.5 & S30W & 1.53 & 0.3 & 0.02 & 1.64 & 0.13 & 0.16 & valley \\
\hline San Fernando & & & N69W & 3.09 & 0.17 & 0.04 & 2.07 & 0.16 & 0.67 & Casta \\
\hline 02.09.1971 & 6.6 & 27.6 & $\mathrm{~N} 21 \mathrm{E}$ & 2.66 & 0.28 & 0.10 & 2.47 & 0.10 & 0.26 & old rid \\
\hline Parkfield & & & W & 2.88 & 0.44 & 0.01 & 1.33 & 0.19 & 0.63 & Parkfie \\
\hline 12.20 .1994 & 5.0 & 9.1 & S & 3.80 & 0.10 & 0.01 & 1.74 & 0.11 & 0.24 & fault \\
\hline Caolinga & & & $\mathrm{W}$ & 2.83 & 0.26 & 0.10 & 2.67 & 0.11 & 0.18 & Cantu \\
\hline 05.02 .1983 & 6.5 & 30.1 & $\mathrm{~N}$ & 2.20 & 0.26 & 0.10 & 2.14 & 0.17 & 0.64 & creek \\
\hline Northridge & & & $\mathrm{S} 74 \mathrm{E}$ & 3.81 & 0.60 & 0.12 & 4.17 & 0.10 & 0.23 & Canog \\
\hline 01.17 .1994 & 6.7 & 5.9 & S16W & 3.43 & 0.34 & 0.09 & 3.50 & 0.09 & 0.34 & park \\
\hline Cape Mendocino & & & W & 3.25 & 0.45 & 0.15 & 2.44 & 0.17 & 0.58 & Petrol \\
\hline 04.25 .1992 & 7.0 & 5.4 & $\mathrm{~S}$ & 2.89 & 0.24 & 0.08 & 2.31 & 0.13 & 0.37 & gener \\
\hline Westmorland & & & $\mathrm{E}$ & 4.35 & 0.33 & 0.11 & 3.26 & 0.13 & 0.27 & Westm \\
\hline 04.26 .1981 & 5.0 & 6.6 & $\mathrm{~S}$ & 3.54 & 0.44 & 0.15 & 3.25 & 0.12 & 0.29 & land $\mathrm{fi}$ \\
\hline Imperial valley & & & S45W & 2.68 & 0.22 & 0.10 & 2.30 & 0.15 & 0.31 & Calexi \\
\hline 10.15 .1979 & 6.4 & 17.4 & N45W & 1.98 & 0.19 & 0.15 & 2.14 & 0.14 & 0.36 & fire \\
\hline
\end{tabular}


Table 2

Nomenclature of constraint scenarios considered

\begin{tabular}{cc}
\hline Case & Constraints imposed \\
\hline 1 & Energy \& PGA \\
2 & Energy, PGA, PGV, \& UBFAS \\
3 & Energy, PGA, PGV, PGD \& UBSAS \\
4 & Energy, PGA, UBFAS \& LBFAS \\
\hline
\end{tabular}

Table 3

Dynamic response of elastic-plastic system for alternative constraint scenarios

\begin{tabular}{ccccc}
\hline Case & 1 & 2 & 3 & 4 \\
\hline$\mu_{\max }$ & 6.58 & 5.12 & 4.89 & 2.86 \\
$u_{\max }(\mathrm{m})$ & 0.4410 & 0.3427 & 0.3276 & 0.1922 \\
$u_{p}(\mathrm{~m})$ & 0.1203 & 0.1032 & 0.0968 & 0.0533 \\
\hline
\end{tabular}


Table 4

Dynamic response of elastic-plastic system for alternative damping ratios, case 4

\begin{tabular}{cccc}
\hline$\zeta$ & 0.02 & 0.03 & 0.05 \\
\hline$\mu_{\max }$ & 3.29 & 2.86 & 2.42 \\
$u_{\max }(\mathrm{m})$ & 0.2208 & 0.1922 & 0.1624 \\
$u_{p}(\mathrm{~m})$ & 0.0718 & 0.0533 & 0.0315 \\
\hline
\end{tabular}

Table 5

Sensitivity analysis of objective function to constraint parameters for inelastic structure, case (4); *: values are calculated at the frequency at which $M_{4}(\omega)$ and $M_{5}(\omega)$ are at respective maxima

\begin{tabular}{ccccccccccc}
\hline Parameter & $E$ & $M_{1}$ & $M_{2}$ & $M_{3}$ & $M_{4}(\omega)$ & $M_{4}(\omega)$ & $\alpha_{1 l}$ & $\alpha_{1 u}$ & $\alpha_{2 l}$ & $\alpha_{2 u}$ \\
\hline$\epsilon_{1}$ & 0.19 & 0.07 & 0.04 & 0.03 & 0.11 & 0.08 & 0.03 & 0.05 & 0.04 & 0.06 \\
$\epsilon_{2}$ & 11.98 & 4.23 & 17.53 & 52.60 & $61.55^{*}$ & $175.33^{*}$ & 112.71 & 69.21 & $17.83^{*}$ & 23.55 \\
\hline
\end{tabular}

